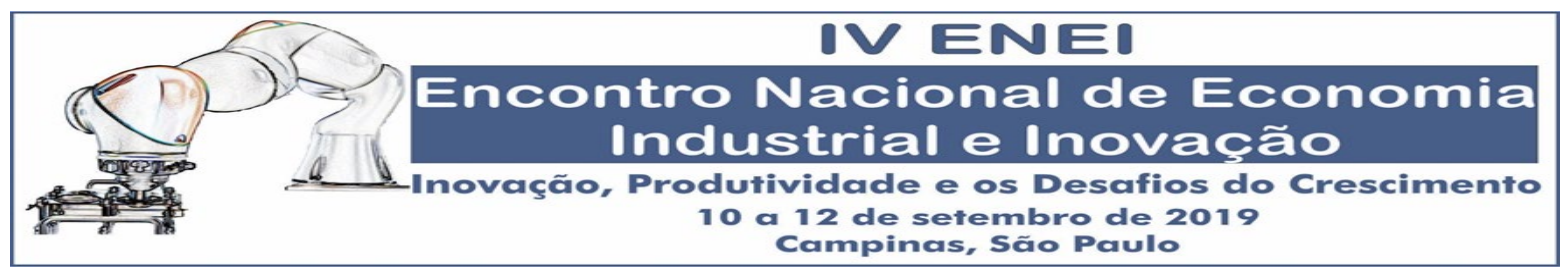

Rotinas de Firmas Industriais Inovadoras do Rio Grande do Sul

\author{
Maria Luísa Lacerda Albertão ${ }^{1}$ \\ Janaína Ruffoni ${ }^{2}$ \\ Fernanda Maciel Reichert ${ }^{3}$
}

\title{
RESUMO
}

As rotinas influentes no processo inovador de firmas industriais são o foco do trabalho. Para identificar o conjunto de rotinas que as firmas com melhor desempenho inovador e econômico executam, foi feita uma análise dos dados de uma survey realizada em 2015, com 1.331 firmas industriais do Rio Grande do Sul. Para esta investigação foram realizadas análises descritivas e bivariadas e a ferramenta de equações estruturais com estimação Partial Least Squares (PLS). O estudo apontou para um conjunto restrito de 41 empresas com desempenho superior à média. Os resultados corroboram com a literatura da teoria econômica evolucionária, informando que há um grupo de firmas que realizam rotinas específicas e possuem desempenho econômico e inovador superior as demais. Esse estudo permite ampliar o conhecimento sobre o comportamento da firma industrial inovadora e refletir a cerca da possiblidade de políticas públicas e privadas voltadas para ampliar o grupo das firmas inovadoras.

Palavras-chave: Rotinas da Firma. Desempenho Econômico e Inovador. Firmas Industriais.

\begin{abstract}
The influential routines in the innovative process of industrial firms are the focus of the paper. To identify the set of routines that the firms with the best innovative and economical performance have, an analysis of the data of a survey applied in 2015, with 1,331 industrial firms of Rio Grande do Sul was carried out. For this investigation, descriptive and bivariate analysis the structural equation tool with Partial Least Squares (PLS) estimation. The study pointed to a small set of 41 top performing firms. The results corroborate with the literature of evolutionary economic theory, informing that there is a group of firms that perform specific routines and have economic and innovative performance superior to the others. This study allows to increase the knowledge about the behavior of the innovative industrial firm and to reflect on the possibility of public and private policies aimed at expanding the group of innovative firms.
\end{abstract}

Key-words: Firm's routines; Economic and Innovative Performance; Industrial Firms.

JEL Code: L20, L6 e O31.

Área ABEIN: 3.4 - Estratégias e gestão da C\&T\&I

\footnotetext{
${ }^{1}$ Mestra em Economia - PPGE/UNISINOS - luisalacerdalb@gmail.com

2 Professora do Programa de Pós-Graduação em Economia - PPGE/UNISINOS - jruffoni@unisinos.br

${ }^{3}$ Professora do Programa de Pós-Graduação em Administração - PPGA/NITEC/UFRGS - fernanda.reichert@ufrgs.br
} 


\section{Rotinas de Firmas Industriais Inovadoras do Rio Grande do Sul}

\section{Introdução}

As discussões acerca da compreensão do progresso tecnológico exigem entendimentos a respeito do processo inovador realizado por um dos principais agentes do sistema de inovação: a firma. A firma é considerada o lócus da inovação na teoria neo-schumpeteriana. Entender como a inovação é realizada e gera resultados é fundamental para avançar nas explicações relativas à mudança tecnológica e seus impactos.

Uma contribuição importante da teoria neo-schumpeteriana ao tentar explicar o comportamento da firma inovadora é o conceito de rotina. Esse conceito é introduzido por Nelson e Winter (1982), o qual apresenta que a rotinização das atividades de uma empresa constitui uma importante forma de geração de conhecimento específico. Esses autores destacam também que a existência de uma atividade inovadora está relacionada às rotinas que a firma realiza. Compreender que um conjunto de rotinas confere resultados superiores no mercado para as firmas é fundamental para avançar no entendimento da dinâmica da mudança tecnológica.

Aprofundar os estudos sobre as rotinas das firmas é relevante, pois ainda existem campos abertos para a pesquisa do tema, tais como a correlação entre rotinas, aprendizado e conhecimento (MILAGRES, 2011). As organizações vivem sobre a égide da inovação, isto é, sob a necessidade de criar novas rotinas ou recombinar rotinas existentes, portanto, cabe questionar qual o papel das rotinas em renovar o conhecimento das organizações. De acordo com D'Adderio et al. (2013), o ambiente cada vez mais incerto e em constante mudança, exige das firmas maior atenção as suas rotinas. Captar o modo como as organizações aprendem a encontrar um equilíbrio entre a estabilidade e coerência, por um lado, e a flexibilidade e mudança, por outro, não é trivial. Tal atividade exige abandonar as visões estáticas da organização para revelar a microdinâmica da inovação, incluindo os processos por meios dos quais as rotinas emergem e evoluem.

Assim, objetiva-se neste estudo elaborar uma análise a respeito da atividade inovativa da firma com base nas suas rotinas. Mais especificamente, pretende-se responder à seguinte pergunta: Qual é o conjunto de rotinas realizadas por firmas inovadoras que as confere desempenho econômico superior no mercado?

Para responder à questão da pesquisa, analisou-se por meio de modelagem de equações estruturais qual o conjunto de rotinas que impactam no desempenho de um grupo de firmas inovadoras de setores industriais do Rio Grande do Sul.

O artigo apresenta, a seguir, uma revisão da literatura a respeito de inovação e rotinas das firmas. Na sequência, apresenta os procedimentos metodológicos adotados no estudo. A análise dos resultados, a partir da avaliação do modelo estrutural, é apresentada na seção 4. Por último, são feitas as considerações finais.

\section{Inovação e rotinas das firmas}

Desenvolver uma teoria evolucionária do comportamento das firmas para a inovação foi o que Nelson e Winter (1982) realizaram. A busca da firma em introduzir mudanças em seus produtos e processos, em um ambiente de seleção de mercado, e, com resultados determinados pelo comportamento das firmas, é explicada pelas suas rotinas. As firmas estão comprometidas com a busca de maneiras de aprimorar seus lucros, mas não é suposto que suas ações sejam maximadoras de lucros em um conjunto de escolhas dadas e bem definidas, e sim, a ideia de uma 'seleção natural' econômica relacionada com a habilidade das firmas de sobreviver e crescer. No centro da análise estará a inovação das empresas, e os lucros são considerados o meio pelo qual as firmas inovadoras bem sucedidas crescem em relação às 
demais. Os autores ressaltam que a concorrência é um processo dinâmico ativo, e que algumas firmas se esforçam deliberadamente para liderar as inovações.

De acordo com Possas (2008), a teoria evolucionária de Nelson e Winter abriu uma nova frente para a análise microeconômica da dinâmica industrial e tecnológica. As firmas com rotinas adequadas à obtenção de maior lucratividade são mais propensas a crescer e lucrar. Segundo Tigre (2005) três princípios podem ser destacados como chaves para entender a teoria evolucionista. O primeiro é que a dinâmica econômica é baseada em inovações em produtos, processos e nas formas de organização da produção. As inovações não são necessariamente graduais, podendo assumir caráter radical, e, neste caso, gerando instabilidade no sistema econômico. O segundo princípio descarta a racionalidade invariante dos agentes econômicos. Os evolucionistas apontam para a necessidade de desenvolver uma visão da firma constituída de indivíduos distintos e dotada de características cognitivas próprias. A diversidade conduz à ideia de racionalidade limitada, ou seja, de que a racionalidade dos agentes não pode ser pré-definida, pois é resultante do processo de aprendizagem ao longo das interações com o mercado e novas tecnologias. O terceiro princípio se refere à propriedade de autorregulação da firma, como resultado das flutuações do mercado, ou seja, um ambiente de incertezas. É rejeitado qualquer tipo de equilíbrio de mercado, conforme proposto pela teoria tradicional, na medida em que não é possível alcançá-lo em ambiente coletivo de flutuações de agentes individuais com rotinas e capacitações distintas.

A teoria evolucionista usou a ideia de seleção natural econômica, onde o sucesso, ou seja, a busca pelo lucro estaria na procura incessante das firmas em introduzir inovações selecionadas pelo mercado. Assim como os genes são características persistentes dos organismos, são hereditários, selecionáveis e determinam seu comportamento, no modelo evolucionário são as rotinas (regras de decisão) que determinam os comportamentos regulares e previsíveis da firma, rotina para a produção de bens, para procedimentos e como estratégias na resolução de problemas não rotineiros. Em outras palavras, as rotinas que importam, são aquelas que se modificam e evoluem na busca e seleção de melhores oportunidades (NELSON; WINTER, 1982).

Para Tigre (2005), a competitividade de uma empresa, em uma atividade particular, é definida pelos evolucionários como um conjunto de competências tecnológicas diferenciadas de ativos complementares e de rotinas. As firmas devem estar preocupadas em resolver e identificar o problema tecnológico, pois não é somente o mecanismo de preço que explica o funcionamento do mercado. É necessário um processo de busca (processo de aprendizagem cumulativa) para introduzir inovações que serão selecionadas pelo mercado.

As firmas tomarão suas decisões de acordo com as rotinas e como elas se modificam ao longo do tempo. As mudanças de rotina ocorridas a partir de esforços para solucionar problemas é o ponto chave para o desenvolvimento de uma nova estratégia, novo produto, novo processo, isto é, a firma usando a rotina de busca para resolver problemas e inovar.

$\mathrm{Na}$ tentativa de ampliar a discussão, foram identificados autores contemporâneos que discutem as 'rotinas da firma' à luz da teoria econômica evolucionária e suas principais contribuições. Para tanto, foi feita uma busca bibliográfica de artigos publicados em periódicos nacionais e internacionais da área, no período de 2000 a 2016, usando as palavras chaves: 'rotinas dinâmicas', 'rotinas organizacionais', 'capacidade de inovação' e 'desempenho inovador'.

As rotinas de busca são atividades associadas às necessidades da firma de se adaptar aos contextos internos e externos da organização. As rotinas mudam com as experiências, contribuindo tanto para a estabilidade quanto para a adaptação. Mudanças de rotina estão associadas aos esforços para solucionar problemas que se iniciam com a rotina existente, estas novas combinações pode ser a fonte para processos de inovação.

A literatura afirma que as rotinas possuem várias características, algumas são repetitivas e coletivas e outras podem ser estáveis e dinâmicas.

Segundo Milagres (2011), embora na literatura existam evidências de que as rotinas promovem estabilidade, há também indicações de que estas mudam como fruto das experiências. Assim, as rotinas podem ser entendidas de uma maneira dinâmica. Para que se encaixem como dinâmicas, devem quebrar o padrão, superar limitações e se adequar às mudanças impostas pela dinâmica competitiva do mercado. 
Com múltiplas repetições, as rotinas tornam-se automáticas, isto é, aumentam o conhecimento e domínio a cerca da operação, com isto os atores tornam-se mais aptos a comparar resultados, entender particularidades, evitar erros e ineficiências e, então, respostas novas podem ser identificadas. Em situações marcadas por inovações, os agentes contam com o imprevisto, em condições de forte incerteza e para problemas complexos buscam por rotinas simplificadoras e, então, aumentam a confiança em padrões adotados, liberam espaços cognitivos e com isso reduzem a incerteza. As rotinas existem porque respondem aos desafios impostos às empresas, entre os quais se destacam coordenação e minimização de conflitos.

Conforme Pentland et al.(2012), na literatura atual, as rotinas são conceituadas como tendo dois níveis (partes, aspectos ou camadas): (1) um nível concreto que consiste nos desempenhos específicos da rotina e podem apresentar variações e; (2) um nível abstrato que tanto molda como é moldado por estes desempenhos concretos. O nível concreto é referido como aspecto "performativo" e o abstrato como o "ostensivo". Referem-se ao nível abstrato simplesmente como "história", ações passadas e atuais podem ser seletivamente retidas como parte da história. Embora possa ser interpretada de muitas maneiras (habilidades, disposições) há dependência de um caminho. Quando não há história todas as ações são igualmente prováveis.

A relação entre os aspectos ostensivos e performativos das rotinas cria uma oportunidade contínua de variação, seleção e retenção de novas práticas e padrões de ação dentro das rotinas e permite que as rotinas gerem um amplo resultado de estabilidade aparente a mudanças consideráveis. (PENTLAND et al., 2012).

A combinação da influência de agentes humanos e artefatos materiais, segundo D'Adderio (2011), podem ser comparados com a ideia de um nível concreto (físico) e abstrato (motivacional). Artefato é qualquer coisa feita pela arte humana e mão-de-obra, algo criado por pessoas normalmente para um propósito prático e está normalmente envolvido no decurso de uma rotina. Artefatos são visto como 'memória externa'.

Artefatos típicos incluem ferramentas, modelos e procedimentos escritos, bem como informações sofisticadas e tecnologia de comunicação. Autores que enfatizam o uso de artefatos, equipamentos físicos e máquinas, têm uma influencia óbvia sobre as rotinas de fabricação, como, por exemplo, novas tecnologias de informação e comunicação que criaram cada vez mais artefatos sofisticados. Conjuntos de rotinas podem ser combinados e recombinados para criar processos. Na literatura tecnológica, os blocos de construção são chamados de "processos" ou "serviços" ao invés de rotinas. (PENTLAND et al.,2012).

Como destacam Lewin et al. (2011), são necessárias rotinas específicas para que a empresa consiga assimilar, transformar e explorar os conhecimentos internos e externos entre os membros e departamentos da empresa.

Milagres (2011) apresenta um quadro no qual foram listados trabalhos que discutem os diferentes papéis das rotinas (Quadro 1).

A rotina é vista como uma capacidade da firma que envolve diversas atividades coordenadas e, também, sua própria mudança, conceituada como rotina dinâmica (envolve busca e seleção, processo de aprendizagem cumulativa de erros e acertos). Inovar exige mudança contínua em uma escala de rotinas usadas em uma firma com o objetivo de criar novos produtos e processos.

Os principais autores contemporâneos abordados na revisão bibliográfica afirmam que as rotinas possuem várias características, algumas são repetitivas e coletivas e outras podem ser estáveis e dinâmicas. Para Becker (2004), as rotinas proporcionam algum grau de estabilidade, proporcionam o necessário para detectar a novidade. É desta forma que as rotinas permitem aos pesquisadores mapear a mudança organizacional e a mudança incremental nas próprias rotinas. 
Quadro 1 - Papéis das rotinas das firmas e alguns estudos

\begin{tabular}{|l|l|l|}
\hline \multicolumn{1}{|c|}{ Papéis } & \multicolumn{1}{|c|}{ Descrição } & \multicolumn{1}{c|}{ Estudos a respeito dos papéis das rotinas } \\
\hline $\begin{array}{l}\text { Oferecem coordenação, } \\
\text { controle e coerência. }\end{array}$ & $\begin{array}{l}\text { As rotinas dão estrutura para } \\
\text { as ações da empresa, } \\
\text { sequências e uniformidade. }\end{array}$ & $\begin{array}{l}\text { Becker (2005); Dosi et al. (2000); Nelson e } \\
\text { Winter (1982); Cohen e Bacdayan (1994); Cyert } \\
\text { e March (1963); Langlois (1992); Narduzzo, } \\
\text { Rocco e Warglien (1997); Knot e McKelvey } \\
\text { (1999); Sherer, Rogovsky e Wright (1998) }\end{array}$ \\
\hline Agem como gatilhos & $\begin{array}{l}\text { Podem ser acionadas e acionar } \\
\text { outras rotinas }\end{array}$ & $\begin{array}{l}\text { Nelson (1994); Betsch, Fiedler e Brinkmann } \\
\text { (1998); Weick (1990); Avery (1996); Cohen e } \\
\text { Bacdayan (1994) }\end{array}$ \\
\hline Minimizam conflitos & $\begin{array}{l}\text { Rotinas mediam questões } \\
\text { ligadas à disputa de poder e } \\
\text { conflitos }\end{array}$ & $\begin{array}{l}\text { Nelson e Winter (1982); Lazarick, Mangolte e } \\
\text { Massué (2000); Inam (1997); Cyert, March e } \\
\text { Simon (1997); Denis e Lazarick (1999, mimeo). }\end{array}$ \\
\hline Reduzem a incerteza & $\begin{array}{l}\text { Simplificam, reduzem a } \\
\text { complexidade das decisões, } \\
\text { aumentam a confiança nos } \\
\text { padrões adotados e, com isso, } \\
\text { diminuem a incerteza. }\end{array}$ & $\begin{array}{l}\text { Becker (2001;1988); Vanberg (1993); Simon } \\
\text { (1979); Hodgson (2000); Dosi et al. (1993); Dosi } \\
\text { e Egidi (1991); Becker e Knudsen (2000, } \\
\text { mimeo); Avery (1996). }\end{array}$ \\
& $\begin{array}{l}\text { Rotinas são a memória das } \\
\text { organizações, lócus do } \\
\text { conhecimento }\end{array}$ & $\begin{array}{l}\text { Nelson e Winter (1982); Dosi, Teece e Winter } \\
\text { (1992); Levitt e March (1988); Cohen e } \\
\text { Bacdayan (1994); Teece e Pisano (1994); Zollo e } \\
\text { Winter (2002); Costello (2000); Pentland (1992); } \\
\text { Denis e Lazarick (1999); Lazaric, Mangolte e } \\
\text { Massué (2000). }\end{array}$ \\
\hline $\begin{array}{l}\text { Incorporam } \\
\text { conhecimento }\end{array}$ & $\begin{array}{l}\text { Becker e Knudsen (2001); Simon (1977); Egidi e } \\
\text { Narduzzo (1997); Egidi (1996); Hegselmann e } \\
\text { Terna; Heidelberg, Springer e Verlag; Ashmos, } \\
\text { Duchon e McDaniel (1998). }\end{array}$ \\
\hline $\begin{array}{l}\text { Reduzem o uso de } \\
\text { recursos cognitivos }\end{array}$ & $\begin{array}{l}\text { Rotinas permitem ações } \\
\text { automáticas e, com isso, } \\
\text { liberam capacidade cognitiva. }\end{array}$ \\
\hline
\end{tabular}

Fonte: MILAGRES (2011.pg. 177).

Segundo Milagres (2011), com múltiplas repetições as rotinas tornam-se automáticas, isto é, aumenta o conhecimento a respeito da operação, tornando os atores mais aptos a comparar resultados e entender particularidades, sendo possível assim evitar erros e ineficiências e propor novas e melhores rotinas.

Para Pentland e Feldmann (2005), a estrutura interna de uma rotina pode produzir uma ampla gama de resultados diferentes, entre "muito estável" e "mudando constantemente", dependendo das circunstâncias.

A compreensão dos papéis que as rotinas desempenham nas firmas é importante. Pode-se afirmar que há um conjunto desses, conforme discutido na literatura e apresentado a seguir:

a) Coordenação e controle: definem um conjunto de ações que as empresas podem acompanhar de maneira satisfatória e guiam a seleção e interpretação de informações recebidas de diferentes fontes (MILAGRES, 2011). Uma linha de base estável para o aprendizado, para ajudar a coordenação de processos (BECKER, 2004). Alguns processos rotineiros (ex.: rotinas de gerenciamento de projetos) são capazes de produzir resultados diferentes. Como exemplo, uma empresa de arquitetura pode usar um processo reconhecível e repetitivo para projetar edifícios, contudo cada projeto tem um resultado diferente. Já há outras rotinas são focadas em produzir exatamente o mesmo. (D'ADDERIO et al., 2013);

b) Trégua: para Pentland e Feldmann (2005), a rotina como ideia de trégua sugere que esta tenha o papel de solução de conflitos. Diferentes indivíduos podem possuir distâncias cognitivas entre si,

\footnotetext{
${ }^{4}$ As citações bibliográficas presentes neste quadro não constam na seção de 'Referências' deste estudo. Trata-se de uma lista bibliográfica construída por Milagres (2011).
} 
como os agentes precisam interagir nas organizações, pode haver um grande potencial de conflito. Mas as rotinas, ao criarem referenciais para ações dos indivíduos, minimizam este potencial. Para lidar com este problema Nelson e Winter (1982) descrevem uma importante característica da rotina que é "propiciar trégua". Rotinas atuam no sentido de medir questões ligadas à disputa de poder e conflitos nas organizações (MILAGRES, 2011). Muitas "formas de fazer as coisas" nas organizações são, em grande medida, resultado de decisões administrativas para desempenhar a tarefa de determinada forma. Nelson e Winter (1982) reformularam este problema de resolução de conflito através do argumento "trégua". (BECKER et al., 2005);

c) Gatilho: as rotinas podem ser acionadas e acionar outras rotinas (MILAGRES, 2011). Alguns elementos podem acionar determinados padrões, como interrupções, feedbacks, experiências antigas, entre outros (BECKER, 2004). A descrição das rotinas organizacionais precisa ser minuciosa para permitir rastrear a dinâmica que estabiliza e desestabiliza os processos.

d) Economia de recursos cognitivos: as rotinas têm o papel de economizar recursos mentais e melhorar a capacidade de decisão. (BECKER, 2004). Rotinas representam o depósito de conhecimentos acumulados pelas empresas e permitem que o que foi absorvido seja realizado de forma automática. Assim, os agentes economizam em recursos cognitivos, abrindo espaço para outros aprendizados (MILAGRES, 2011). As rotinas reduzem a carga cognitiva e opera através de procedimentos de memória. (D'ADDERIO et al., 2013);

e) Redução da incerteza: a incerteza coloca problemas na tomada de decisões. Para lidar com a incerteza, a estratégia é aumentar a quantidade de informações buscadas e tratadas; o aumento da rotinização serve para reduzir a incerteza na tomada de decisão. (BECKER, 2004). Rotinas advêm da necessidade de os agentes resolverem problemas em contextos marcados por incerteza. (MILAGRES, 2011);

f) Armazenamento de conhecimentos: a rotinização da atividade em uma organização constitui a parte mais importante de forma de armazenagem do conhecimento operacional específico. Entende-se que o conhecimento pode ser estocado, aplicado e alterado. As rotinas criam linguagem comum, que permite à organização compartilhar, criar e analisar o conhecimento. As rotinas apresentam um elo importante para a compreensão do aprendizado organizacional, especialmente no que se refere à codificação do conhecimento tácito. (MILAGRES, 2011).

No quadro 2, afim de sistematizar o conteúdo visto até o momento, são apresentadas algumas definições de rotinas.

Quadro 2 - Resumo de algumas discussões a respeito do conceito de rotina

\begin{tabular}{|l|l|}
\hline \multicolumn{1}{|c|}{ Autoria } & \multicolumn{1}{c|}{ Discussões a respeito de rotina } \\
\hline Jones e Craven (2000) & $\begin{array}{l}\text { A gestão se preocupa em grande medida com as rotinas que lidam com novas } \\
\text { situações. Tais rotinas não são repetitivas, mas são executadas sem pensamento } \\
\text { consciente detalhado. Não são fáceis de adquirir, pois representam o que a } \\
\text { empresa aprendeu ao longo do tempo por meio de um processo de tentativa e } \\
\text { erro. }\end{array}$ \\
\hline Becker (2004) & $\begin{array}{l}\text { Caracterizada por apresentar regularidade e padrão de comportamento que é } \\
\text { seguido repetidamente, mas está sujeito a mudaças caso as condições se } \\
\text { alterarem. }\end{array}$ \\
\hline Becker et al (2005) & $\begin{array}{l}\text { Rotinas são quase sempre um fluxo. Algumas rotinas mudam mais rapidamente } \\
\text { e mais drasticamente do que outras. Por outro lado, algumas rotinas podem ser } \\
\text { estáveis (ex.: gestão de inventário, formação de preços, processo de } \\
\text { recrutamento). Consideram dois níveis de observação de rotinas, um nível } \\
\text { concreto para o padrão de ação corrente e um nível abstrato para o desempenho } \\
\text { das pessoas. }\end{array}$ \\
\hline $\begin{array}{l}\text { Pentland, Feldman } \\
\text { (2005) }\end{array}$ & $\begin{array}{l}\text { Podem ser caracterizadas como padrões abstratos, onde os participantes as } \\
\text { usam para orientar o desempenho. As rotinas são vistas como um aspecto } \\
\text { performativo }\end{array}$ \\
\hline
\end{tabular}




\begin{tabular}{|l|l|}
\hline D'Adderio (2007) & $\begin{array}{l}\text { A combinação de agentes humanos e artefatos materiais podem ser comparados } \\
\text { com a ideia de um nível concreto (físico) e um nível abstrato (motivacional). } \\
\text { Artefatos como "memória externa", ajudam a compartilhar parte da carga } \\
\text { cognitiva. }\end{array}$ \\
\hline Milagres (2011) & $\begin{array}{l}\text { Capacidade do ator em repetir uma ação, podendo ser coletiva quando estão } \\
\text { distribuídas ao longo da organização. Rotinas são persistentes, o que promove } \\
\text { especialização, coerência e minimiza conflitos. As rotinas são dinâmicas } \\
\text { quando atendem às necessidades da empresa de se adaptar aos contextos } \\
\text { interno e externo. }\end{array}$ \\
\hline Pentland et al (2012) & $\begin{array}{l}\text { Rotinas são conceituadas como tendo dois níveis (partes, aspectos, camadas). } \\
\text { Um nível concreto que consiste no desempenho específico da rotina e podem } \\
\text { apresentar variações. E um nível abstrato que tanto molda como é moldada por } \\
\text { estes concretos desempenhos O nível concreto é referido como aspecto } \\
\text { "performativo" o abstrato como aspecto "ostensivo". O nível abstrato pode ser } \\
\text { interpretado de muitas maneiras (habilidades, disposições, história) e existe a } \\
\text { dependência de um caminho. Quando não há história, todas as ações são } \\
\text { igualmente prováveis. }\end{array}$ \\
\hline $\begin{array}{l}\text { Wohlgemuth, Wenzel } \\
\text { (2015) }\end{array}$ & $\begin{array}{l}\text { Há diferentes efeitos da rotinização, um nível mais dinâmico no nível } \\
\text { estratégico e uma rotinização mais estática no nível operacional. }\end{array}$ \\
\hline
\end{tabular}

Fonte: Elaboração própria a partir de Milagres (2011)

Considerando esses diferentes papéis das rotinas da firma, vale retomar que neste trabalho o objetivo é compreender qual é o conjunto de rotinas que se destacam na geração da inovação e no desempenho econômico de um conjunto específico de firmas industriais.

A seguir são apresentados os procedimentos metodológicos.

\section{Procedimentos metodológicos}

O presente estudo foi realizado a partir da análise de dados secundários advindos de uma pesquisa survey realizada por um projeto de pesquisa acadêmica que tinha por objetivo mapear a inovação na indústria gaúcha. Tal projeto, realizado com as empresas industriais do Estado do Rio Grande do Sul, foi realizado entre 2010 e 2015 , teve envolvimento de quatro universidades e teve apoio financeiro de agências de fomento públicas. O questionário foi aplicado a uma base de dados de 6.142 empresas, constituída a partir do recorte das que tinham cinco ou mais funcionários, do universo de 10.930 registros da Federação das Indústrias do Rio Grande do Sul (FIERGS) de 2010. Foram respondidos, ao todo, 1.331 questionários válidos. O questionário continha questões com respostas de múltipla escolha e em escala do tipo Likert (com cinco níveis de concordância). Foram selecionadas, de um total de 62 questões, 42 questões (Quadro 3) que tinham relevância para avaliar as "rotinas" das firmas, o desempenho econômico, o desempenho inovador e informações gerais.

Quadro 3- Questões para avaliação das rotinas da firma

\begin{tabular}{|l|l|}
\hline \multicolumn{1}{|c|}{ Questões de escala tipo Likert ( 5 pontos de concordância): } \\
\hline$>$ Realiza a concepção original dos seus próprios produtos. \\
\hline$>$ Monitora as últimas tendências tecnológicas do setor. \\
\hline$>$ Utiliza metodologias formais de gestão de projetos (Stage-Gate, PMBOK, Funil da inovação, etc) \\
\hline$>$ Adapta as tecnologias em uso para suas necessidades. \\
\hline$>$ Realiza prototipagem de seus produtos. \\
\hline$>$ Desenvolve produtos em parcerias com ICTs. \\
\hline$>$ Lança seus próprios produtos.
\end{tabular}


Formaliza os procedimentos de PCP .

Mantém controle estatístico do processo.

Utiliza equipamentos atualizados na fronteira tecnológica.

Realiza o processo produtivo conforme programado.

$>$ Estabelece uma rotina produtiva que não gera retrabalho.

$>$ Consegue expandir a capacidade instalada sempre que necessário.

$>$ Define formalmente seus objetivos estratégicos anualmente.

$>$ Integra todos seus setores com o uso de informática.

$>$ Padroniza e documenta os diferentes procedimentos de trabalho.

$>$ Atualiza suas técnicas e ferramentas de gestão.

$>$ Mantém a capacitação de pessoal adequada para as diferentes funções da empresa (treinamento).

$>$ Utiliza práticas modernas de gestão financeira.

$>$ Realiza pesquisas formais para monitorar o mercado.

$>$ Realiza pesquisas para medir a satisfação de seus clientes.

$>$ Utiliza critérios formais para a seleção de seus fornecedores.

\section{Relativas ao desempenho econômico:}

O lucro líquido vem crescendo de forma contínua nos últimos três anos.

O percentual de participação da empresa do mercado vem crescendo de forma contínua nos últimos 3 anos.

$>$ O faturamento da empresa vem crescendo de forma contínua nos últimos três anos.

Questões de múltipla escolha:

Disparo de desenvolvimento:

Como o desenvolvimento acontece?

A programação da produção é feita segundo:

$>$ A tomada de decisão está condicionada:

$>$ As mais recentes melhorias relacionadas à gestão ocorreram:

O principal foco da gestão é:

Quanto ao modelo de Gestão, pode-se dizer que:

Onde acontecem as principais mudanças relacionadas à área comercial:

$>$ Levando em conta sua empresa, quais são as três opções que, de fato, caracterizam inovação para ela?

$>$ Enumere as áreas em ordem de ocorrência da maior parte das novidades de sua empresa (sendo que 1 é onde mais acontecem e 4 onde menos acontecem mudanças).

Questões de informações gerais:

Número aproximado de funcionários?

Qual o faturamento bruto da empresa em 2013?

Qual o percentual de investimento em Pesquisa e Desenvolvimento no faturamento bruto da empresa?

$>$ Qual a data de fundação da empresa?

\section{Relativas ao desempenho inovador:}

$>$ Qual o total de patentes registradas na empresa?

$>$ Qual o número de novos produtos lançados em 2013?

$>$ Qual o percentual de faturamento decorrente de novos produtos lançados em 2013?

Fonte: Elaboração própria com base no questionário original do projeto de pesquisa acadêmica 'Caminhos da Inovação da Indústria Gaúcha'.

A análise descritiva das perguntas selecionadas foi desenvolvida a partir dos dados obtidos pelas frequências das respostas, utilizando a totalidade da amostra ( $\mathrm{n}=1331$ empresas). As estatísticas estão baseadas em todos os casos com dados válidos. Foi utilizado o sistema SPPS (Statistical Package for Social Science, versão 2.1).

Após análise, foi necessário identificar as firmas com desempenho inovador e econômico superior e procurar criar um grupo de empresas representativo. 
A partir da resposta da questão sobre como se dá o disparo do desenvolvimento de novos produtos, tecnologias ou processos nas firmas observou-se que há um grupo de empresas que agem de forma reativa, realizando o desenvolvimento apenas quando solicitado por clientes ou por exigências legais. Por outro lado, existe um grupo menor de empresas que se apresenta de forma mais proativa, realizando desenvolvimentos com o propósito de apresentar novidades para o mercado que foram planejadas, projetadas e desenvolvidas pela própria firma, ou seja, por iniciativa própria.

Conforme a Tabela 1, a análise do grupo identificado no presente trabalho como proativas se torna importante quando o tema é inovação e suas rotinas. São 203 empresas que representam 15,3\% da amostra total.

Tabela 1 - Análise das empresas conforme disparo do desenvolvimento

\begin{tabular}{lccl}
\hline & $\begin{array}{c}\text { Quantidade de } \\
\text { empresas }\end{array}$ & $\begin{array}{c}\text { \% do total } \\
\text { pesquisado }\end{array}$ & Grupo \\
\hline Solicitação do cliente & 504 & 37,9 & Reativa \\
Melhoria de produto existente & 468 & 35,2 & Reativa \\
Aumento do portfólio de produtos & 162 & 12,2 & Proativa \\
Atendimento requisitos legais & 153 & 11,5 & Reativa \\
Invenção & 41 & 3,1 & Proativa \\
Ausente & 3 & 0,2 & \\
\hline TOTAL & 1331 & 100 & \\
\hline
\end{tabular}

Fonte: Elaboração própria com base nos resultados do software SSPS.

As empresas proativas são na sua maioria pequenas e microempresas (faturamento anual inferior a R \$16 milhões), de baixa e médio-alta intensidade tecnológica, mais da metade são dos setores de Couro e Calçados, Máquinas e Equipamentos, Alimentos, Plástico e Borrachas, Móveis, Produtos de Metal e Vestuário. O principal modelo de gestão é de organização familiar profissionalizada, diferentemente do grupo das empresas reativas que tem o modelo de gestão personalizada, centralizada na figura do(s) proprietário(s). Verifica-se, assim, a importância de uma gestão profissionalizada.

Para todas as questões de escala tipo Likert foi utilizado o teste-t, com base na significância estatística das diferenças de médias entre as empresas reativas e proativas. A partir dos resultados encontrados foi possível selecionar as rotinas com diferencial e o desempenho econômico das empresas proativas.

A análise bivariada baseada em tes-t é utilizada para rejeitar ou não uma hipótese, para uma média de dados pareada tipo Likert. Conforme Hair et al. (2005) a probabilidade de atingir significância estatística é baseada não apenas em considerações estatísticas, mas também na verdadeira magnitude do efeito de interesse. O p-valor é a probabilidade de se obter uma estatística de teste igual ou mais extrema que aquela observada em uma amostra, o valor terá significância estatística para valores iguais ou menores que $5 \%$,ou seja, para nível de confiança igual ou maior que 95\%. Então para um valor de $\mathrm{p}<0,05$, pode-se concluir que o efeito reflete as características para a população.

Foram feitas análises das questões relacionadas ao desempenho econômico dessas 203 firmas proativas em relação às demais empresas, e, apenas 41 empresas tiveram média superior (Tabela 2) e se destacaram por obter um resultado estatisticamente significativo na questão relativa ao crescimento regular nos últimos três anos do percentual de participação da empresa no mercado, evidenciando que empresas inovadoras são as que lançam novos produtos no mercado e assim conseguem aumentar seu marketshare.

Este grupo formado por 41 empresas que responderam que o disparo de desenvolvimento se dá por invenção (Tabela 1) se destacam por relatar a realização de uma atividade relativamente rara para o conjunto de firmas respondentes, ou seja, inovar com base em iniciativa própria e apresentando 
desempenho econômico superior. Por esta razão, serão identificadas no presente trabalho como inovadoras.

Tabela 2 - Desempenho econômico das empresas inovadoras

\begin{tabular}{llccc}
\hline \multicolumn{1}{c}{ Estatística do grupo } & & $\mathrm{N}$ & Dif. Média & $\mathrm{p}$ valor \\
\hline $\begin{array}{l}\text { O lucro líquido da empresa vem crescendo de } \\
\text { forma contínua nos últimos três anos (LLC) }\end{array}$ & Inovadores & 41 & 0,123 & 0,444 \\
\cline { 2 - 5 } & Demais & 1283 & & \\
\hline $\begin{array}{l}\text { O percentual de participação da empresa no } \\
\text { mercado vem crescendo de forma regular nos } \\
\text { últimos três anos (PPM) }\end{array}$ & Inovadores & 40 & 0,417 & $\mathbf{* 0 , 0 0 2}$ \\
\cline { 2 - 5 } & Demais & 1280 & & \\
\hline $\begin{array}{l}\text { O faturamento da empresa vem crescendo de forma } \\
\text { contínua nos últimos três anos (FTC) }\end{array}$ & Inovadores & 41 & 0,168 & 0,235 \\
\cline { 2 - 5 } & Demais & 1280 & & \\
& & & & \\
\end{tabular}

\begin{tabular}{l}
\hline * Valores significantes $\mathrm{p}<0,050$ \\
Fonte: Elaboração própria com base nos resultados do software SSPS
\end{tabular}

Na sequência, analisou-se o conjunto de rotinas executados por estas empresas em comparação às demais. Para verificar se este grupo possui rotinas estatisticamente significativas, foi realizado o test-t nas questões de escala tipo Likert relativas às rotinas (Quadro 1). Todas tiveram média acima das demais empresas e 15 foram estatisticamente significativas (Tabela 3 ).

As rotinas numeradas e com um indicador no final são as que se destacam neste grupo de empresas inovadoras e as demais são aquelas rotinas que não se diferenciam das demais empresas do RS.

Verifica-se que as empresas inovadoras realizam a concepção original de seus produtos, monitorando as últimas tendências tecnológicas do setor e adaptando as tecnologias em uso para suas próprias necessidades, realizam a prototipagem e também efetuam treinamentos a fim de manter a capacitação do pessoal adequada para as diferentes funções da empresa. É possível verificar um conjunto de rotinas de busca necessárias para a capacidade de desenvolvimento, rotinas para absorver e transformar uma dada tecnologia, rotinas que representam o depósito de conhecimentos acumulados pelas empresas e firmando o papel das rotinas em armazenar conhecimento. A partir de sua compreensão pode-se entender como o conhecimento é estocado, aplicado, deteriorado e, consequentemente, alterado.

Este grupo de empresas por formalizarem seus procedimentos de PCP, confirmam a utilização de rotinas operacionais para planejar, preparar, programar e executar suas operações diárias. Em sua gestão, integram todos os setores com o uso da informática, padronizam e documentam os diferentes procedimentos de trabalho. Observado o uso de rotinas necessárias para realizar a tarefa de coordenar de forma eficiente as atividades internas. Papel das rotinas de controle e coordenação, dando regularidade, unidade e sistematicidade as práticas de um grupo. Mais padronizado, mais fácil de comparar, mais fácil de controlar. Guiam a seleção e interpretação de informações recebidas de diferentes fontes.

As empresas inovadoras lançam seus próprios produtos, utilizam equipamentos atualizados na fronteira tecnológica e conseguem expandir a capacidade instalada sempre que necessário, e então acionar novas rotinas de trabalho. As rotinas podem ser acionadas e acionar outras rotinas, alguns elementos podem acionar determinados padrões. Como, por exemplo, ao lançar produtos, a firma necessita levar seu produto em escala comercial até o mercado e com isto adquiri recursos externos. Recursos externos no sentido de captar informações de mercado, de mudança de demanda, o que pode desencadear novos processos internos para a firma, novas rotinas. Ao usar equipamentos atualizados poderá obter uma nova rotina de processo, um novo padrão. Verifica-se o papel de rotina de gatilho. 
Tabela 3 - Conjunto de rotinas das empresas inovadoras

\begin{tabular}{lrr}
\multicolumn{1}{c}{ Teste T - Amostras independentes } & Dif.média & *p valor \\
\hline 1. Realiza concepção original dos seus próprios produtos (COP) &, 546 &, 000 \\
2. Monitora as últimas tendências tecnológicas do setor (MTT) &, 605 &, 000 \\
3. Utiliza metodologias formais de gestão de projetos (MGP) &, 649 &, 000 \\
4. Adapta as tecnologias em uso para as suas próprias necessidades (ATN) &, 550 &, 000 \\
5. Realiza a prototipagem de seus produtos (RPT) &, 499 &, 002 \\
Desenvolve produtos em parceria com ICT's &, 342 &, 145 \\
6. Lança seus próprios produtos (LPP) &, 896 &, 000 \\
7. Formaliza o procedimento de PCP (FPP) &, 505 &, 002 \\
8. Mantém controle estatístico do processo (CEP) &, 381 &, 017 \\
9. Utiliza equipamentos atualizados na fronteira da tecnologia do setor (EFT) &, 366 &, 008 \\
Realiza processo produtivo conforme o programado &, 093 &, 357 \\
Estabelece uma rotina produtiva que não gera retrabalho &, 185 &, 082 \\
10. Consegue expandir a capacidade instalada sempre que necessário (ECI) &, 477 &, 002 \\
Define formalmente seus objetivos estratégicos &, 022 &, 869 \\
11. Integra todos seus setores com o uso de informática (ISI) &, 459 &, 000 \\
12. Padroniza e documenta os diferentes procedimentos de trabalho (PDP) &, 338 &, 010 \\
Atualiza suas técnicas e ferramentas de gestão &, 276 &, 054 \\
13. Mantém a capacitação de pessoal adequada (CPA) &, 241 &, 041 \\
14. Utiliza práticas modernas de gestão financeira (PGF) &, 392 &, 011 \\
15. Realiza pesquisas formais para monitorar o mercado (PMM) &, 686 &, 000 \\
Realiza pesquisa para medir a satisfação de seus clientes &, 238 &, 178 \\
Utiliza critérios formais para a seleção de seus fornecedores &, 185 &, 252 \\
\hline \hline *Valores significantes p < 0,050 & & \\
Fonte: Elaboração própria a partir dos resultados do software SSPS & & \\
& &
\end{tabular}

Utilizam metodologias formais de gestão de projetos, realiza pesquisas formais para monitorar o mercado, mantêm controle estatístico do processo e práticas modernas de gestão financeira. Papel das rotinas de reduzir incertezas, a incerteza coloca problema da tomada de decisões. Para lidar com a incerteza a estratégia é aumentar a quantidade e qualidade das informações

Dando continuidade a análise das rotinas utilizadas pelo grupo de empresas inovadoras, foram selecionadas algumas frequências das respostas para as questões de múltipla escolha.

Com relação ao processo de desenvolvimento de produto, grande parcela das empresas inovadoras $(40,6 \%)$ afirma que o desenvolvimento acontece fragmentado em meio às atividades rotineiras da empresa. Tal dado chama a atenção para o objeto de estudo, pois evidenciam a existência de uma relação entre a operação rotineira e a memória da organização, rotinas como lócus do conhecimento (NELSON E WINTER,1982). As rotinas que lidam com novas situações não são repetitivas, são executadas sem pensamento consciente detalhado e não são fáceis de adquirir porque representam o que a empresa aprendeu ao longo do tempo através de um processo de tentativa e erro.

O principal foco da gestão das empresas inovadoras é o de melhoria contínua, e, as mais recentes melhorias ocorreram na infraestrutura administrativa (base física e equipamentos) e nos sistemas, técnicas e ferramentas utilizadas. O papel destas rotinas é garantir a sobrevivência das empresas em ambientes dinâmicos, adaptando seu conhecimento e práticas. Novo conhecimento está relacionado com uma nova rotina, chamadas de rotina dinâmica ou mutante, mudam como fruto das experiências/aprendizado. Para se encaixarem como rotinas dinâmicas, elas devem quebrar o padrão e estão ligadas a busca e seleção.

Conforme análise inicial da base de dados foi possível verificar, conforme já está claro na literatura, que há uma relação entre rotinas e desempenho econômico e rotinas e desempenho inovador. Apesar de estar claro na literatura, só se justifica se os impactos positivos no desempenho econômico da firma puderem ser comprovados, para tanto o estudo se propôs não apenas analisar as rotinas das empresas inovadoras, mas também entender quais são os efeitos entre rotinas e desempenho econômico. 
Para verificar como o desempenho pode ser explicado por rotinas selecionadas, foi utilizada a modelagem de equações estruturais utilizando a técnica Partial Least Squares ${ }^{5}$, a qual permite representar múltiplas relações simultaneamente a partir do uso de variáveis latentes (RADOSEVIC; YORUK, 2013). $\mathrm{O}$ procedimento caracteriza-se por permitir que diversas variáveis sejam correlacionadas simultaneamente, traçando o comportamento dos diferentes fatores envolvidos no objeto de análise (HAIR ET AL. 2005).

O modelo estrutural (Figura 1) apresenta a relação de causalidade entre o conjunto de rotinas e o desempenho econômico. O conjunto de rotinas será entendido como o papel da rotina conforme fundamentação teórica.

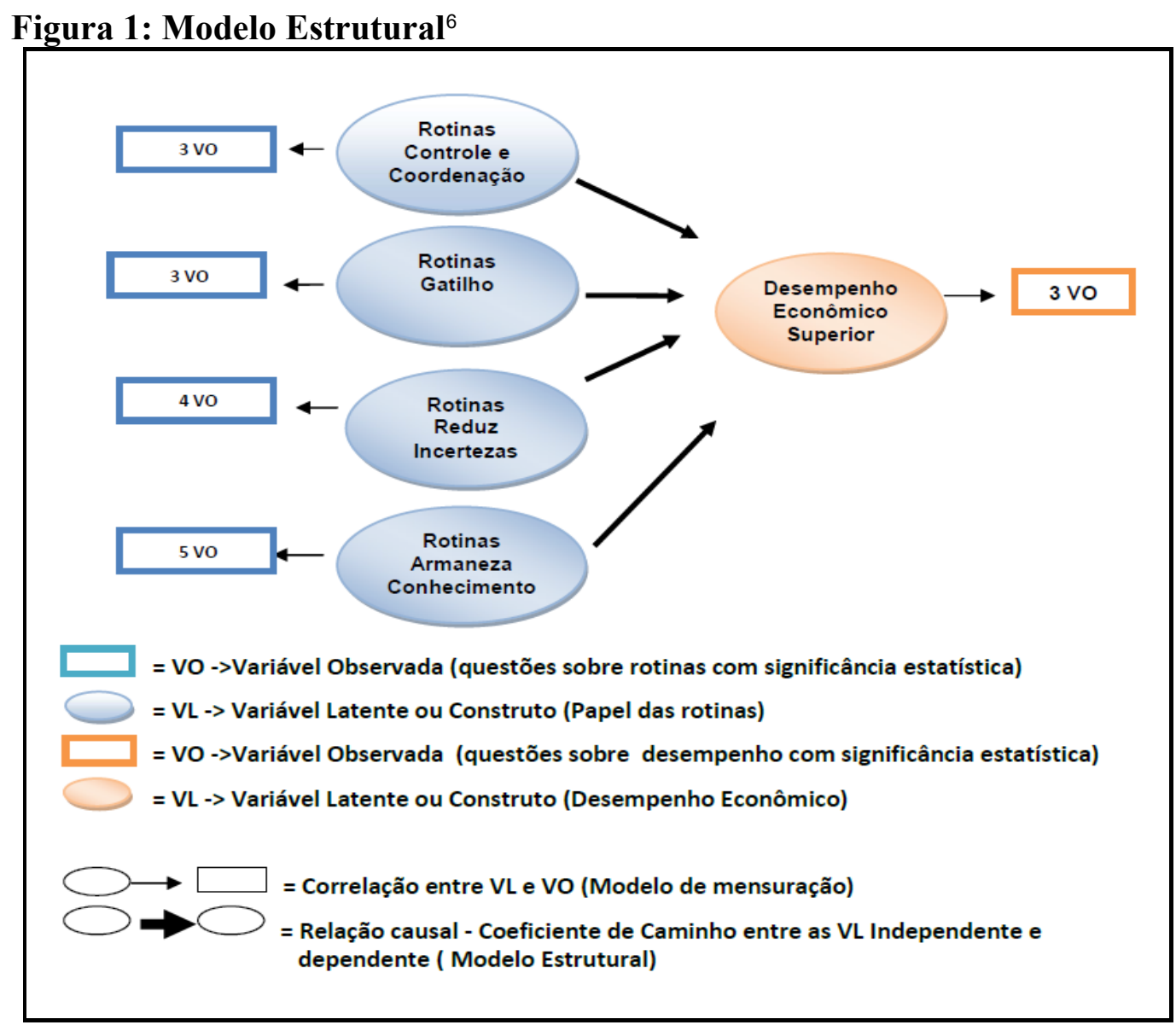

Fonte: Elaboração própria com base em Ringle, Silva e Bido (2014)

Recomenda-se avaliar os níveis de validade e confiabilidade do modelo de mensuração antes de testar o modelo estrutural (Fornell; Larcker, 1981; Koufteros, 1999; Hair et al., 2009, Ringle et al., 2014) para especificar os indicadores em cada construto (Hair et al., 2009). .

As cargas fatoriais de cada fator (variável latente) foram avaliadas para definir o modelo estrutural final a ser testado. Para verificar se todas as rotinas compreendidas nos quatro papéis seriam agrupadas corretamente, foi empregada a análise fatorial numa perspectiva confirmatória e observou-se que os indicadores "lança seus próprios produtos", "realiza pesquisas formais para monitorar o mercado" e "monitora as últimas tendências tecnológicas" não atenderam aos parâmetros mínimos exigidos $(\geq 0,7)$, (segundo Hair et.al 2014, os valores de 0,6 a 0,7 são considerados o limite inferior de acessibilidade) e foram eliminadas. O modelo foi testado novamente e um indicador, "adapta as tecnologias em uso para suas próprias necessidades", ainda apresentou valor inferior ao ponto de corte de 0,7 , no entanto, por estar

\footnotetext{
${ }^{5}$ A partir do uso do software SmartPLS, versão 3.2.7.

${ }^{6}$ No modelo estrutural, o papel das rotinas de 'economizar recursos cognitivos' e de 'trégua' não foram incluídos por serem referentes ao comportamento das pessoas, um nível mais abstrato do papel das rotinas, de difícil mensuração.
} 
muito próximo desse valor e por ser importante para o modelo teórico, optou-se por mantê-lo sem prejuízo aos testes de confiabilidade. Ressalta-se que Hair et al. (2009) aceitam valores a partir de 0,6 e Kim et al. (2013) usaram um ponto de corte de 0,5 nas cargas fatoriais. A Tabela 4 mostra o modelo final.

Tabela 4 - Análise fatorial confirmatória

\begin{tabular}{|c|c|c|c|c|c|c|c|c|c|c|}
\hline & \multicolumn{2}{|c|}{$\begin{array}{l}\text { Armazenamento } \\
\text { Conhecimento }\end{array}$} & \multicolumn{2}{|c|}{$\begin{array}{c}\text { Controle e } \\
\text { Coordenação }\end{array}$} & \multicolumn{2}{|c|}{ Gatilho } & \multicolumn{2}{|c|}{$\begin{array}{r}\text { Redução } \\
\text { Incertezas }\end{array}$} & \multicolumn{2}{|c|}{$\begin{array}{c}\text { Desempenho } \\
\text { Econômico }\end{array}$} \\
\hline & \multicolumn{2}{|c|}{ Iteração } & \multicolumn{2}{|c|}{ Iteração } & \multicolumn{2}{|c|}{ Iteração } & \multicolumn{2}{|c|}{ Iteração } & \multicolumn{2}{|c|}{ Iteração } \\
\hline & 1 & 2 & 1 & 2 & 1 & 2 & 1 & 2 & 1 & 2 \\
\hline ATN & 0,673 & 0,694 & & & & & & & & \\
\hline COP & 0,801 & 0,782 & & & & & & & & \\
\hline CPA & 0,769 & 0,731 & & & & & & & & \\
\hline RPT & 0,686 & 0,710 & & & & & & & & \\
\hline MTT & 0,562 & - & & & & & & & & \\
\hline FPP & & & 0,776 & 0,776 & & & & & & \\
\hline ISI & & & 0,766 & 0,766 & & & & & & \\
\hline PDP & & & 0,724 & 0,724 & & & & & & \\
\hline $\mathrm{ECl}$ & & & & & 0,868 & 0,876 & & & & \\
\hline EFT & & & & & 0,943 & 0,953 & & & & \\
\hline LPP & & & & & 0,381 & - & & & & \\
\hline CEP & & & & & & & 0,784 & 0,801 & & \\
\hline MGP & & & & & & & 0,908 & 0,915 & & \\
\hline PGF & & & & & & & 0,724 & 0,850 & & \\
\hline PMM & & & & & & & 0,444 & - & & \\
\hline FTC & & & & & & & & & 0,879 & 0,879 \\
\hline LLC & & & & & & & & & 0,906 & 0,906 \\
\hline PPM & & & & & & & & & 0,719 & 0,719 \\
\hline
\end{tabular}

Fonte: Elaboração própria com base SmartPLS.

Nota: os itens em destaque foram excluídos do modelo em função da baixa carga fatorial.

A Tabela 5 traz os indicadores avaliados para validade e confiabilidade do modelo e demonstra que todos os valores são adequados. A confiabilidade de cada constructo foi testada pelo Alfa de Cronbach, sendo que todos os valores apresentaram valores acima de 0,6 (Hair et al., 2014). A confiabilidade composta (CR) também foi testada, com o intuito de avaliar as escalas. Os valores foram maiores que 0,7 (Hair et al., 2014; Kim et al., 2013; Radosevic; Yoruk, 2013). A variância média extraída (AVE) demonstrou que todos os valores foram acima de 0,5 (Fornell; Larcker, 1981; Fang et al., 2014; Hair et al., 2009; Henseler et, al 2009; Ringle et al., 2014).

Tabela 5 - Confiabilidade e validades dos construtos

\begin{tabular}{lccc}
\hline & Alfa de Cronbach & CR & AVE \\
\hline Desempenho Econômico & 0,783 & 0,876 & 0,703 \\
Rotinas de Armazenamento de Conhecimento & 0,715 & 0,822 & 0,537 \\
Rotinas de Controle e Coordenação & 0,632 & 0,800 & 0,571 \\
Rotinas de Gatilho & 0,816 & 0,912 & 0,838 \\
Rotinas de Redução de Incertezas & 0,817 & 0,892 & 0,734 \\
\hline
\end{tabular}

Fonte: Elaboração própria com base SmartPLS.

Por fim, a Tabela 6 apresenta a validade discriminante para o modelo final, conforme indicado por Fornell e Larcker (1981), onde as raízes quadradas dos valores das AVEs de cada variável latente devem ser maiores do que as correlações (de Pearson) entre as variáveis latentes. 
Tabela 6 - Validade discriminante

\begin{tabular}{|c|c|c|c|c|c|}
\hline & $\begin{array}{l}\text { Desempenho } \\
\text { Econômico }\end{array}$ & $\begin{array}{c}\text { Rotinas } \\
\text { Armazenar } \\
\text { Conhecimento }\end{array}$ & $\begin{array}{c}\text { Rotinas } \\
\text { Controle e } \\
\text { Coordenação }\end{array}$ & $\begin{array}{l}\text { Rotinas } \\
\text { Gatilho }\end{array}$ & $\begin{array}{c}\text { Rotinas } \\
\text { Reduz } \\
\text { Incertezas }\end{array}$ \\
\hline Desempenho Econômico & 0,839 & & & & \\
\hline Rotinas - Armazenar Conhecimento & 0,491 & 0,733 & & & \\
\hline Rotinas - Controle e Coordenação & 0,581 & 0,500 & 0,756 & & \\
\hline Rotinas - Gatilho & 0,317 & 0,596 & 0,659 & 0,915 & \\
\hline Rotinas - Reduz Incertezas & 0,357 & 0,603 & 0,595 & 0,686 & 0,857 \\
\hline
\end{tabular}

Fonte: Elaboração própria com base SmartPLS.

Alcançados os patamares mínimos requeridos, comprova-se que os indicadores utilizados em cada variável latente são fortemente inter-relacionados e o modelo é consistente e confiável em sua mensuração (Hair et al., 2005).

\section{Análise dos resultados: avaliação do modelo estrutural}

Uma vez realizadas as medidas de validação dos construtos do modelo, testou-se o modelo estrutural proposto na Figura 1. O modelo pressupõe que os quatro papéis das rotinas ("Armazenamento de Conhecimento", "Controle e Coordenação", "Gatilho" e "Redução de Incertezas") estão relacionados positivamente com o desempenho das firmas inovadoras.

O modelo estrutural foi avaliado levando-se em consideração os coeficientes dos caminhos e o valor de $\mathrm{R}^{2}$. Os caminhos são testados usando teste-t (Kim et al., 2013) e, ao analisar os valores de significância $(\mathrm{p} \leq 0,05)$, as relações positivas entre as rotinas de "Armazenamento de Conhecimento" e "Controle e Coordenação" e o desempenho mostraram-se significativas. As relações entre as rotinas de "Gatilho" e de "Redução de Incertezas" não foram significativas e, portanto, não foram suportadas. A Tabela 7 mostra essas relações.

Tabela 7-Resultado relações entre rotinas e desempenho econômico

\begin{tabular}{r|lrrrr}
\hline & & $\begin{array}{c}\text { Coeficiente } \\
\text { Estrutural }\end{array}$ & Estatística T & Valores de P* \\
\hline H1 & $\begin{array}{l}\text { Controle e Coordenação -> Desempenho } \\
\text { Econômico }\end{array}$ & 0,596 & 3,170 & 0,002 & Suportado \\
H2 & $\begin{array}{l}\text { Gatilho -> Desempenho Econômico } \\
\text { Redução Incertezas -> Desempenho }\end{array}$ & $-0,276$ & 1,168 & 0,243 & Não suportado \\
H3 & $\begin{array}{l}\text { Econômico } \\
\text { Armazenamento Conhecimento -> }\end{array}$ & $-0,037$ & 0,177 & 0,860 & Não suportado \\
H4 & \begin{tabular}{l} 
Desempenho Econômico \\
\hline
\end{tabular}
\end{tabular}

*significância para $\mathrm{p} \leq 0,05$

Fonte: Elaboração própria com base em SmartPLS.

As rotinas de 'armazenamento de conhecimento' tratam do entendimento de como o conhecimento é estocado, aplicado, deteriorado e, consequentemente alterado. Criam linguagem comum, que permite à organização compartilhar, criar e analisar o conhecimento. Servem como memória da organização, configurando-se lócus de acumulação de conhecimento. Representam soluções bemsucedidas para resolver problemas. (NELSON E WINTER, 1982). Assim, aquelas firmas que conseguem ter rotinas para adaptar as tecnologias em uso conforme suas necessidades, que realizam a concepção original dos seus próprios produtos, que mantém a capacitação de pessoal adequada para as diferentes funções da empresa e que realizam prototipagem dos seus próprios produtos conseguem armazenar conhecimento de forma a impactar positivamente no desempenho da firma. Atividades como essas, 
permitem que as firmas se antecipem ante seus concorrentes, pois seu conhecimento permite que se diferenciem das demais.

Da mesma forma, aquelas firmas que possuem rotinas bem desenvolvidas para formalizar os procedimentos de planejamento e controle da produção, para integrar seus setores com uso de informática e para padronizar e documentar os diferentes procedimentos de trabalho, conseguem atingir melhor desempenho. A formalização das atividades rotineiras que envolvem a operação da empresa é a essência para o estabelecimento de rotinas. As rotinas de 'controle e coordenação' são aquelas que dão regularidade, unidade e sistematicidade às práticas de um grupo. Oferecem referências à ação dos indivíduos e coordenam suas atividades. As rotinas de controle e coordenação definem um conjunto de ações que as empresas podem acompanhar e guiam a seleção e interpretação de informações recebidas de diferentes fontes.

Surpreendentemente, as rotinas de 'gatilho' e de 'redução de incertezas' não levam essas empresas a atingir desempenho superior. Isso posto, observa-se um papel tímido daquelas rotinas voltadas às atividades de operação da empresa, como atualização dos equipamentos, expansão da capacidade instalada, controle estatístico de processos, gestão de projetos. Ou seja, as rotinas mais voltadas ao desenvolvimento de produtos e à gestão parecem ter mais impacto do que aquelas rotinas mais voltadas à atividade operacional do dia-a-dia. Essa constatação corrobora com os achados de Alves et al. (2017), que identificaram um grupo de capacidades dinâmicas que afetam positivamente o desempenho da empresa, que são justamente aquelas capacidades que contam com rotinas voltadas às atividades de desenvolvimento de novos produtos e de tecnologias, bem como às atividades de gestão e transação. Complementarmente, os autores identificaram uma capacidade ordinária que não afeta diretamente o desempenho inovador das firmas. Essa capacidade conta, justamente, com aquelas rotinas relacionada à operação das firmas.

Assim, esses resultados apontam para o que foi demonstrado no modelo estrutural. O poder de explicação do modelo estrutural é avaliado pelo valor de $\mathrm{R}^{2}$ da variável dependente (Hair et al., 2009; Kim et al., 2013). No presente estudo, o valor de $\mathrm{R}^{2}$ do desempenho como variável dependente das rotinas das firmas foi de 0.43 . Isso significa que as rotinas das firmas explicam mais de $40 \%$ do desempenho econômico das firmas. Existem outros mais de 50\% representando outros fatores que podem impactar nesse desempenho, como aspectos institucionais e ambientais, setor de atuação e porte da empresa, nível tecnológico, entre outros.

Assim, o modelo estrutural de rotinas influentes no desempenho econômico das firmas está apresentado na Figura 3.

Figura 3: Modelo de Desempenho Econômico das Empresas Inovadoras

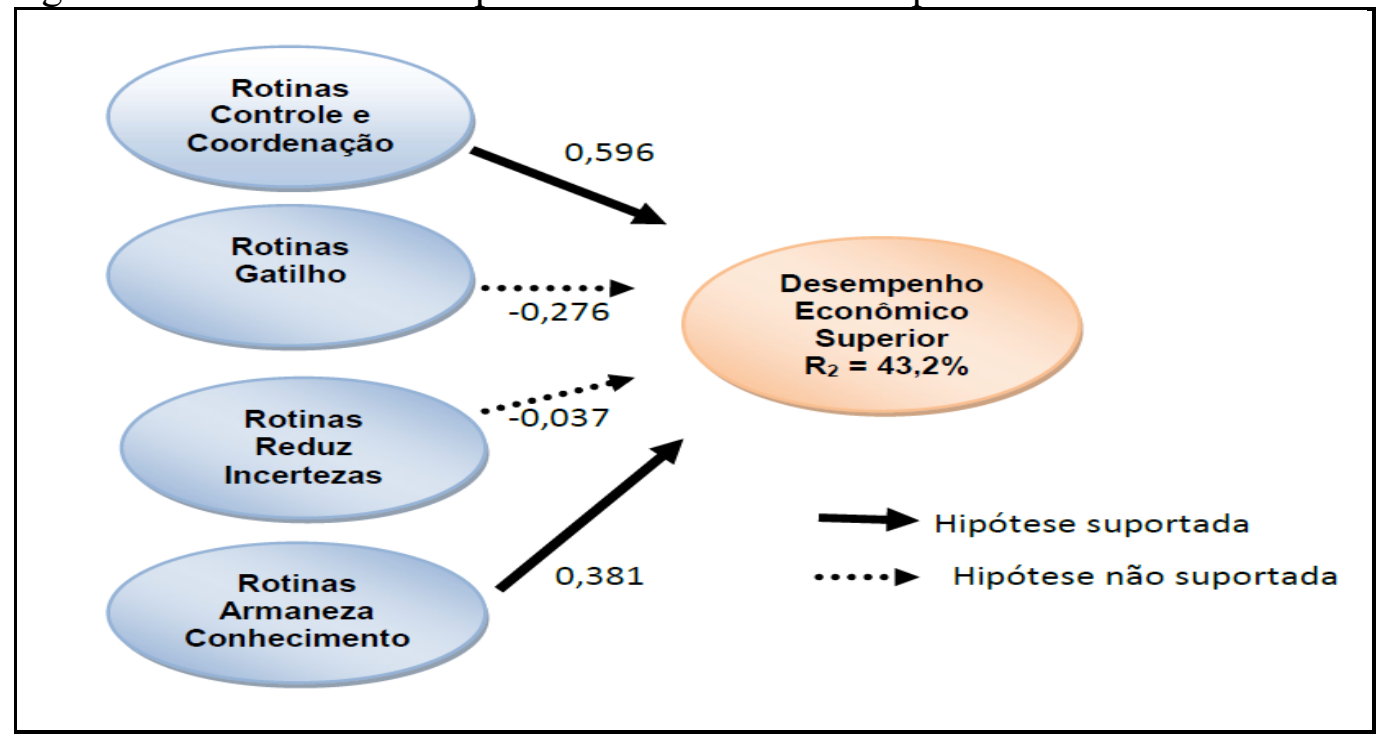

Fonte: Elaboração própria com base SmartPLS. 
O modelo resultante do estudo confere importância do fator interno 'rotina' como elemento diferenciador do desempenho de firmas inovadoras. Assim, se a inovação é uma forma que as firmas encontram de fornecer respostas a um ambiente em constante mudança, seus fatores internos, aqui estudados pelas 'rotinas', são elementos explicativos deste comportamento inovador.

\section{Considerações Finais}

O trabalho realizado contribui com a discussão na área da microeconomia evolucionária ao demonstrar que existe relação entre determinadas rotinas realizadas pela firma, conforme Nelson e Winter (1982) e o seu desempenho econômico.

A partir da análise de 41 firmas de setores industriais do Rio Grande do Sul identificadas como inovadoras, buscou-se verificar qual é o conjunto de rotinas realizadas por firmas industriais inovadoras que as confere desempenho superior no mercado. Para tanto, selecionou-se quatro papéis que as rotinas podem desempenhar: 'armazenamento de conhecimento'; 'controle e coordenação'; 'gatilho'; e redução de incertezas'. Esses papéis formaram o modelo testado pelo método com estimação Partial Least Squares (PLS).

Os resultados mostraram que apenas dois papéis das rotinas influenciam na obtenção de um desempenho econômico superior, as rotinas de 'armazenamento de conhecimento' e as de 'coordenação e controle'.

As rotinas relacionadas ao 'armazenamento de conhecimento' são aquelas voltadas ao monitoramento, adaptação e desenvolvimento de novas tecnologias. Acompanhando esses elementos, está um indicador importante, que é a manutenção da capacidade do pessoal envolvido nessas atividades. Este indicador é crucial na transformação de uma informação (por exemplo, de que existe uma nova tecnologia no setor) em resultados para a empresa (por exemplo, o desenvolvimento de uma nova tecnologia ou de um novo produto que permita que a firma gerasse um desempenho superior). Essas rotinas permitem que, por meio do compartilhamento de conhecimento, a firma consiga lidar, da melhor forma possível, com as mudanças.

As rotinas de 'coordenação e controle' vêm na forma de complementar o conhecimento armazenado. Uma vez que a firma passa a lidar com uma nova tecnologia ou um novo produto, ela precisa de outras rotinas para mantê-lo em funcionamento. São elas que reduzem as incertezas de cada atividade, uma vez que estabelecem processos e procedimentos padronizados para as diferentes atividades da firma. Assim, rotinas que visem à padronização e formalização das atividades, bem como o acompanhamento das mesmas, passam a ter um papel importante na garantia da estabilidade das novas atividades.

Assim, para atingir desempenho superior as firmas precisam de rotinas que permitam que acompanhem e desenvolvam novas tecnologias e produtos e de rotinas que permitam que essas novidades entrem nas suas operações do dia a dia.

Os resultados deste trabalho contribuem para reflexões acerca de implicações gerenciais, uma vez que permite que os gestores tenham maior conhecimento sobre como as suas rotinas se configuram e quais podem gerar maior retorno para a firma. A partir da análise das rotinas, as firmas podem identificar novos procedimentos de como farão a busca para alterar, aprimorar, ou mesmo analisar as diferentes possibilidades para a mudança visando à inovação. As firmas utilizam diferentes rotinas, com maior ou menor grau de sucesso. Não existem receitas gerais, mas sugestões para rotinas mais eficazes que estão relacionadas com a dinâmica inovativa de firmas industriais.

O trabalho também contribui para reflexões de políticas públicas voltadas com foco na firma, seu viés inovador e sua capacitação em rotinas específicas. As rotinas têm uma das chaves para a compreensão da mudança econômica e para entender como as capacidades são acumuladas, transferidas e aplicadas, como a novidade emerge em um sistema baseado em rotinas.

Este estudo limitou-se a analisar um número relativamente baixo de empresas, todas elas localizadas em uma mesma região brasileira. A expansão do estudo pode contribuir para reforçar o 
modelo e oferecer explicação para aqueles conjuntos de rotinas que não se destacaram como fatores relevantes no desempenho das firmas.

\section{Referências Bibliográficas}

ALVES, M.F.R; ALVES, A.F.R.; JUNIOR, H.A.S; GALINA, S.V.R; Cognition in Organizational Routines Research: a Systematic Review. Anais do II ENEI, 2017

BECKER, M.C. Organization routines: a review of the literature. Industrial and Corporate Change. Vol.13(4), pp.643-677, 2004.

BECKER, M.C; LAZARICK, N; NELSON R. R; WINTER, S, G. Applying organizational routines in understandig organizational change. Industrial and Corporate Change.Vol.14, Number 5, pp.775-791, 2005.

CORIAT, B; DOSI, G. Learning how to govern and learning how to solve problems: on the co-evolution of competences, conflict, and organizational routines. International Institute for Applied System Analysis. IIASA Working Paper, WP-95-006, February, 1995.

D'ADDERIO, L. The performativity of routines: Theorizing the influence of artefacts and distributed agencies on routines dynamics. Institute for the Study of Science Technology and Innovation (ISSTI). University of Edinburgh. September, 2007.

D'ADEERIO, L. Artifacts at the centre of routines: Performing the material, turn in routines theory. Journal of Institutional Economics. V. 7:2, 197-230, 2011.

D'ADEERIO, L; FELDMAN, M; LAZARIC, N; PENTLAND, B. Special Issue on Routines Dynamics: Exploring Sources of Stability and Change in Organizations. Organization Science. Sep., 2013.

FANG, Y, .et.al. Trust, satisfaction, and online purchase intention: the moderating role of perceived effectiveness of e-commerce institutional mechanisms. MIS Quartely, 38 (2), 407-247, 2014.

FORNELL, C. \& LARCKER, D. F. Evaluating structural equation models with unobservable variables and measurement error. Journal of Marketing Research,18(1), 39-50, 1981

GOTZ, O.; LIEHR-GOBBERS, K. e KRAFFT, M. Evaluation of structural equation models using the partial least squares (PLS) approach. In: Vinzi, V.E.; Chin,W.W.; Henseler,J. Wang, H. (editors). Handbook of partial least squares. Heidelberg: Springer, 2010.

HAIR, J.F.; BLACK, W.; BABIN, B.; ANDERSON, R.; TATHAM, R. Análise Multivariada de Dados. 5. ed., Porto Alegre: Bookman, 2005.

HAIR, J.F.; BLACK, W.; BABIN, B.; ANDERSON, R.; TATHAM, R. Análise Multivariada de Dados. 6. ed., Porto Alegre: Bookman, 2009.

HAIR, J.F.; HUlt, T.M.; RINGLE, C.M. e SARStedT, M. A Primer on Partial Least Squares Structural Equation Modeling (PLS-SEM). Los Angele: SAGE 2014.

HENSELER, J.: RINGLE, C.M.; SINKOVICS, R. R. The use of partial least squares path modeling in international marketing. Advances in International Markerting. V.20, p. 277-319, 2009. 
JONES, O; CRAVEN, M. Beyond the routine, innovation management and the teaching company sheme. Aston Business School. April, 2000.

KIM,Y.J.; LEE,J.M.; KOO, C.7 NAM,K. The role of governance effectiveness in explaining IT outsourcing performance. International Journal of Information Management, 33, 850-860, 2013

KOUFTEROS,X. Testing a model of pull production: a paradigm for manufacturing research using structural equation modelling. Journal of Operations Management, 17(4), 467-488, 1999

LEWIN, A; MASSINI, S; PEETERS, C: Microfoundations of internal and external absorptive capacity routines. Journal of Organization Science, p. 81-98, 2011.

MILAGRES, ROSILÉIA. Rotinas: uma Revisão Teórica. Revista Brasileira de Inovação [1677-2504], vol:10 iss:1 jan/jun pp:161 -196, 2011.

NELSON, R.; WINTER, S. (1982). Uma teoria evolucionária da mudança econômica. Campinas, SP: Editora da UNICAMP (para tradução brasileira), 2005.

PENTLAND, B. T; FELDMAN, M. S. Organizational routines as a unit of analysis. Industrial and Corporate Change, Vol.14, Number 5, pp.793-815, 2005.

PENTLAND, B; FELDMAN, M; BECKER, M; LIU, P. Dynamics of organizational routines: a generative model. Journal of Management Studies 49:8. December, 2012.

POSSAS, M. L. Economia Evolucionária Neo-Schumpeteriana: elementos para uma integração micromacrodinâmica. Estudos Avançados, v. 22(63), p. 281-305, 2008.

RADOSEVIC, S. \& YORUK, E. Entrepreneurial propensity of innovation systems. Research Policy, 42, 1-15-1038, 2013.

REICHERT, F. M.; CAMBOIM, G. F.; ZAWISLAK, P. A. Capacidades e trajetórias de inovação de empresas brasileiras. Revista de Administração Mackenzie. São Paulo. Vol. 15, n. 5 (2015), p. 161-194, 2015.

RINGLE, C.M; da SILVA, D.; BIDO, S. Modelagem de equações estruturais com utilização do SMARTPLS. Revista Brasileira de Marketing, v.13, n.2, p56-73, 2014.

TIGRE, P.B. Paradigmas Tecnológicos e Teorias Econômicas da Firma. Revista Brasileira de Inovação. Jan-Jun 2005, Vol 4 (1), pp.187-222

WOHLGEMUTH, V; WENZEL, M. Dynamic Capabilities and Routinization. Journal of Business Research, Vol:69 iss:5pg 1944-1948, 2016. 